\title{
GESCHENKE UND ABGABEN IN DER MYKENISCHEN PALASTKULTUR*
}

\author{
Diamantis Panagiotopoulos (Heidelberg)
}

Die Zersplitterung der griechischen Landschaft in zahlreiche, voneinander klar abgegrenzte geographische Regionen förderte im 2. Jt. v.Chr. die Entwicklung von mehreren souveränen Königtümern, die wirtschaftlich autarke Einheiten bildeten. Die mykenischen Palastzentren des 14. und 13. Jhs. v.Chr., in denen das Erbe des minoischen Palastsystems fortlebte, stellten kleinformatige politische Gebilde dar, deren Selbsterhaltung auf einer - verglichen mit den orientalischen Großstaaten - minimalen Territorialbasis beruhte. Die vorhandenen epigraphischen und archäologischen Zeugnisse zu den mykenischen politischen und ökonomischen Strukturen mögen verhältnismäßig gering sein, doch erlauben sie interessante Einblicke in die Gesetzmäßigkeiten des institutionalisierten Gebens in einer antiken Hofgesellschaft. Im mykenischen Griechenland hat es keine Tribute im eigentlichen Sinne des Wortes gegeben, da die Palastzentren eben keine Territorialstaaten waren, die fremde Völker politisch kontrollierten und ökonomisch ausbeuteten. Daher wird sich im folgenden unser analytischer Blick auf Geschenke und Abgaben beschränken, zwei Typen sozioökonomischen Handelns, die zugegebenermaßen kaum miteinander in Beziehung gebracht werden können. Sie bilden nicht einmal ein antithetisches Paar einer freiwilligen und unfreiwilligen Gabe, da auch bei Geschenken das Element der Freiwilligkeit durch die vorherrschende Ethik des Austausches sehr oft ausscheidet. ' Vorliegender Beitrag erhebt daher keinen Anspruch auf die Behandlung einer inhaltlich kohärenten Thematik, sondern setzt als sein Primärziel die Zusammenstellung von Materialien zu antiken (Ab-)Gabenformen, die im Mittelpunkt dieses interdisziplinären

* Vorliegender Beitrag ist Teil einer umfangreichen Untersuchung zu den Strukturen der mykenischen Palastwirtschaft, die durch ein Forschungsstipendium der Deutschen Forschungsgemeinschaft ermöglicht wurde.

$1 \mathrm{Zu}$ den diversen Verbindlichkeiten des zeremoniellen Austausches im 2. Jt. v.Chr. s. u.a. Zaccagnini, Scambio; ders., in: Carruba et al., Studi Orientalistici, S. 189ff; ders., in: Rowlands et al., Centre and Periphery, S. 57ff. Zum theoretischen Diskurs über die Begriffe der ,Gabe“ und des ,Schenkens"s. zuletzt Wagner-Hasel, Der Stoff der Gaben, S. $27 \mathrm{ff}$. 
Workshops stehen. Da die Möglichkeiten und Grenzen jedes interpretatorischen Ansatzes grundlegend von der Überlieferungslage bestimmt sind, wird folgende Betrachtung mit einer kurzen Darstellung unseres Kenntnisstandes eingeleitet. Die Behandlung der einschlägigen textlichen Zeugnisse konzentriert sich auf die Ebene der historischen Realität mit dem Ziel, die verschiedenen Ausprägungen des Gebens (und Nehmens) als förmlichen Akt möglichst präzis zu fassen und voneinander zu differenzieren. ${ }^{2}$ Abschließend wird der Frage nachgegangen, ob die durch primäre Quellen belegten Geschenk- oder Abgabenformen Niederschlag in der Bilderwelt dieser Periode gefunden haben, und wenn ja, unter welchen Umständen.

\section{ÜBERLIEFERUNGSLAGE}

Als wichtigstes Quellenkorpus der mykenischen Palastadministration gelten die Linear B-Täfelchen, administrative Texte in einer Frühform des Griechischen, die in feuchten Ton eingeritzt wurden. Bislang sind uns ca. 5730 Täfelchen bekannt, die sich auf sechs verschiedene mykenische Palastzentren verteilen. ${ }^{3}$ Die zwei wichtigsten Sammlungen stammen aus Knossos und Pylos - sie machen etwa 92\% des gesamten Bestands aus. Wie aussagekräftig ist dieses Material für unsere Fragestellung? Es ist seit langem bekannt, daß die Linear B-Texte nur einen begrenzten Einblick in die mykenische Wirtschaft und Administration ermöglichen, da sie den Zweck eines temporären ,Zwischenspeichers' ${ }^{6}$ administrativer Informationen erfüllten. ${ }^{4}$ Sämtliche Aufzeichnungen beziehen sich auf das ,laufende‘ Verwaltungsjahr. Dieser enge zeitliche Horizont wäre allerdings kein großes Problem gewesen, wenn die Schreiber alle administrativen Aktivitäten des Palastes detailliert dokumentiert hätten. Dies war allerdings nicht der Fall. Die Schreiber zeigten nur ein selektives Interesse an bestimmten Bereichen oder Prozeduren der Palastökonomie. Grundsätzlich gilt, daß die mykenische Tontafel-Bürokratie nicht das gesamte wirtschaftliche Geschehen im Territorium eines Palastzentrums

${ }^{2}$ Da unser Hauptaugenmerk der säkularen Sphäre der mykenischen Gesellschaft gilt, sind hier Gaben an Götter völlig ausgeklammert, auch wenn sie manchmal faktisch oder terminologisch mit Formen des profanen Gebens eng vergleichbar sind.

${ }^{3}$ Bartoněk, Handbuch, S. 70.

+ Zum temporären Charakter der Linear B-Tontafeln s. Heubeck, Schrift, S. 46; ders., Frïhgriechische Lineartafeln, S. 10; Palaima, in: Laffineur/Niemeier, Politeia, S. 629f. Ferner Driessen, in: Minos 29-30, 1994 95, S. 244, der sie als pre-archives bezeichnet. 
aufzeichnete, sondern nur diejenigen Angelegenheiten, welche eine direkte Relevanz für palatiale Interessen hatten. ${ }^{5}$ Aber auch die Sphäre der palatialen Aktivitäten wurde nicht lückenlos erfaßt, denn einige bedeutende Wirtschaftsfaktoren blieben völlig ausgeblendet. Am häufigsten hat man hier das Fehlen jeglicher Angaben zu Handelsaktivitäten des Palastes mit anderen Zentren inner- und außerhalb der Ägäis bedauert. ${ }^{6}$ Dieses Fehlen kann nicht nur zufallsbedingt sein. Ebenso unwahrscheinlich ist es, daß die mykenischen Paläste nicht über ihre intensiven Handelskontakte Buch führten. Alles spricht dafür, daß ein großer Teil der administrativen Handlungen auf Medien aus vergänglichen Materialien festgehalten wurde, die uns heute nicht erhalten sind. ${ }^{7}$ Die Linear B-Tontafeln erweisen sich damit als Akten einer Kanzlei für interne ökonomische Angelegenheiten der Paläste, die für einen kurzfristigen Gebrauch angelegt waren, die Kartei eines ,Ministeriums des Inneren'. Aber auch in diesem Teilbereich der Wirtschaft scheint sich der Focus der Tontafeln nur auf einige ökonomische Kategorien zu konzentrieren. Der Palast führte mit bürokratischer Akribie vor allem über zwei ökonomische Handlungen Buch: a) seine Ausgaben, in erster Linie Rationen, Verteilung von Rohstoffen an die Palastwerkstätten zur Weiterverarbeitung usw., aber auch Inventarlisten der in den Magazinen befindlichen palatialen Waren und b) die Erfüllung von ökonomischen Verpflichtungen der abhängigen Bevölkerung, und zwar hauptsächlich die verschiedenen Abgabenformen. Zusammenfassend läßt sich festhalten, daß die lückenhafte Überlieferungslage und der selektive Charakter der aufgezeichneten Informationen keine umfassende Rekonstruktion des mykenischen Administrationssystems erlauben.

\section{Diplomatische GeschenKe}

Wegen der engen Perspektive der bürokratischen Erfassung durch die Linear B-Täfelchen erfahren wir von diesem Medium nichts über die sicherlich existierenden Außenbeziehungen eines Palastes mit anderen

${ }^{5}$ Palaima, in: Ilievski/Crepajac, Tractata Mycenaea, S. 259; Halstead, in: Voutsaki/ Killen, Economy and Politics, S. $38 \mathrm{f}$.

${ }^{6} \mathrm{~S}$. Killen, in: Davies/Duhoux, Linear B, S. 265ff. Zu einer Zusammenstellung der spärlichen indirekten Zeugnisse s. Olivier, in: Minos 31-32, 1996-1997, S. 275ff.

S. Heubeck, Schrift, S. 46; Panagl, in: Hänsel, Handel, Tausch und Verkehr, S. 49; ferner Driessen, in: Deger-Jalkotzy et al., Floreant Studia Mycenaea, S. 209; ders., Chariot Tablets, S. 14. 
ägäischen oder außerägäischen politischen Einheiten. ${ }^{8}$ Diplomatische Geschenke werden daher nicht dokumentiert, und es ist äußerst fraglich, ob zukünftige Funde diese negative Beweislage ändern werden. Die Einbindung der mykenischen Zentren in ein weitreichendes Beziehungsgeflecht sozialer Eliten, die Preziosen als eine Art ,symbolischer Währung' austauschten, kann dennoch mit Hilfe orientalischer Quellen belegt werden. Die geringe Anzahl der relevanten Zeugnisse - bisher sind uns nur drei Dokumente bekannt - kann dabei keineswegs ihre Aussagekraft schmälern. Der erste Beleg stammt aus der frühen Phase der mykenischen Kultur und ist somit etwa 250 Jahre älter als der zeitliche Horizont der Linear B-Täfelchen. In den ,Annalen“ Thutmosis' III. sind im Bericht des 42. Regierungsjahres die Geschenke des Landes Tanaja an den ägyptischen König registriert:

[Gaben des, Großen'] von $7 j-n z-j]$

Silber: ein $33 w 3 b i j$-Krug in der Machart der Kfjwe

Gefäße von Eisen, die Henkel von Silber

macht (zusammen Silbergewicht) 56 Deben, 3 Kite. ${ }^{9}$

Das Land $T j-n j-\ddot{j}$ (Tanaja), das sechsmal in ägyptischen Quellen des Neuen Reiches auftaucht, ${ }^{10}$ läßt sich mit an Sicherheit grenzender Wahrscheinlichkeit in der Ägäis und konkreter auf dem griechischen Festland lokalisieren. Hier bieten sich zwei Möglichkeiten an: Tanaja könnte entweder eine allgemeine geographische Bezeichnung für das gesamte Territorium des griechischen Festlands oder der Name eines bestimmten mykenischen Fürstentums gewesen sein. Letzteres erscheint nach dem jetzigen Kenntnisstand plausibler. ${ }^{11}$ Die Zuverlässigkeit und der somit hohe historische Wert dieser Zeilen resultiert aus dem besonderen dokumentarischen Charakter der ,Annalen', der insbesondere in den ,statistischen' Abschnitten des Textes Ausdruck findet, welche lange Listen von Geschenken und Tributen aus nicht-ägyptischen Territorien enthalten. ${ }^{12}$ Der oder die Verfasser der Inschrift hatten offenbar Einsicht in Akten des Palastes bzw. der Schatzverwaltung, wic aus den präzisen

'S. Killen, in: Risch/Müllenstein, Colloquium Mycenaeum, S. 176ff.; Aravantinos, in: Deger-Jalkotzy et al., Floreant Studia Mycenaea, S. $72 f$.

Urk. IV 733,4 8; Lehmann, in: Latacz, Homer-Forschung, S. 109; Cline, International Trade, S. 114, A.32. Das angegebene Silbergewicht der Gefäße entspricht einer Menge von über $5 \mathrm{~kg}$.

If Cline, ebd., S. $114 \mathrm{ff}$.

1 S. Latacz, Troia und Homer, S. 160ff., der für eine Identifizierung mit dem Königtum von Mykene plädiert.

12 S. hierzu Panagiotopoulos, in: ÄL 10, 2000, S. 147. 
und - noch wichtiger - realistischen Mengenangaben der abgelieferten Waren zu erkennen ist. ${ }^{13}$ Das ,Tagebuch des Palastes s sowie ein nicht näher definiertes Dokument im Schatzhaus werden sogar in den ,Annalen' explizit erwähnt. ${ }^{14}$

Die besondere historische Tragweite des ,Annalen'-Passus aus der Sicht der ägäischen Archäologie ist bisher nicht adäquat gewürdigt worden. ${ }^{15}$ Der Beleg für einen Geschenkaustausch zwischen einem mykenischen Lokalherrscher und dem ägyptischen König gewinnt enorm an Bedeutung, weil er aus einer Zeit stammt, aus der mykenische Paläste oder sonstige administrative Strukturen auf dem griechischen Festland unbekannt sind. Die mykenische Kultur ist uns in der Regierungszeit Thutmosis' III. (SH II-III A) nahezu ausschließlich durch Grabfunde vertraut. Das Knüpfen von diplomatischen Beziehungen mit Ägypten seitens eines mykenischen Fürstentums setzt allerdings ein bereits fortgeschrittenes Niveau politischer bzw. administrativer Organisation voraus und spricht für ein Zentrum, das, nachdem es seine Machtstellung innerhalb der Ägäis gefestigt hatte, sich nach außen öffnete und den Kontakt mit der ägyptischen Großmacht suchte. Historisch läßt sich der ,Annalen'-Passus offensichtlich in die Periode des ersten Auftauchens der Mykener in der internationalen politischen Szene des östlichen Mittelmeers einbetten, in der sie möglicherweise das minoische Kreta ablösten, eine Hochkultur, die in der gleichen Zeit infolge einer Reihe von massiven Zerstörungen ihrer Palastzentren niederging. Die qualitative Bezeichnung der Kanne als ein Produkt in der Machart der $K f j w$ (Keftiu $=$ Minoer) läßt sich natürlich wunderbar in das historische Bild dieser Periode einfügen, als die mykenische Kunst unter einem sehr starken minoischen Einfluß stand.

Näher zum chronologischen Horizont der Linear B-Täfelchen stehen zwei weitere Belege, die aus den hethitischen Tontafelarchiven von Hattuša stammen. Sie beziehen sich auf Geschenke des Königs von Ahbijawa, einem geographischen Begriff, der für das mykenische Griechenland oder ein politisches Zentrum innerhalb dieser Region stand. ${ }^{16}$ Auch in diesem Fall ist es wahrscheinlicher, daß damit ein bestimmtes Fürstentum auf dem griechischen Festland gemeint war. In letzter Zeit verdichten sich die Indizien, daß dieses Fürstentum Theben

\footnotetext{
13 Ebd., S. 147 Anm. 95.

1. Urk. IV 693,1 1; 694,7 8.

15 Zu einer Ausnahme s. Lehmann, in: Latacz, Homer-Forschung, S. 109

16 Zur Abhijawa-Problematik s. zuletzt Hope Simpson, in: BSA 98, 2003, S. 203f
} 
in Böotien war. ${ }^{17}$ Beim ersten Beleg handelt es sich um den bekannten Tawagalawa-Brief, dessen Absender der hethitische König Hattušili III. und dessen Empfänger offensichtlich der König von Abbijawa war. ${ }^{18}$ Der hethitische König beschwert sich darin, daß der Gesandte des mykenischen Königs bei seinem letzten Besuch am hethitischen Hof keinen Gruß und kein Geschenk mitgebracht hat:

But when [my brother's messenger] arrived at my quarters, he brought me no [greeting] and [he brought] me no present... ${ }^{19}$

Hattušili III. war der Absender eines zweiten Briefes, in dem ebenfalls von einer Geschenksendung des Königs von Abhijawa die Rede ist. ${ }^{20}$ Dieser Passus läßt sich wegen des fragmentarischen Erhaltungszustandes des Brieftextes nicht schlüssig interpretieren. Der Empfänger des Briefes, vermutlich der König von Arzawa, hatte sich beim hethitischen König nach einem Geschenk des Königs von Ahhijawa erkundigt, das offensichtlich ihm selbst galt. Hattušilis Antwort lautete folgendermaßen:

Concerning the gift of the king of Ahbijawa about which you wrote to me, I do not know how the situation is and whether his messenger has brought anything or not...2!

Auch wenn man die Gründe, die diesen Brief veranlaßten, nicht nachvollziehen kann, gewinnt man einen weiteren Beleg für die Teilnahme des mykenischen Königs von Abhijawa an dem internationalen Beziehungsgeflecht des diplomatischen Geschenkaustausches. Mario Liverani vermutet hier eine Austauschkette, nämlich ein Geschenk des Königs von Abhijawa an Hattušili, der dieses an den König von Arzawa weiterschenkte. ${ }^{22}$ Ein solches Zirkulationsmuster, das es in der Spätbronzezeit sicherlich gegeben hat, läßt sich dieser Lesung des Briefes allerdings nicht mit Sicherheit entnehmen.

17 S. Latacz, Troia und Homer, S. 15lff.; Niemeier, in: Die Hethiter, S. 295. Zu alternativen Vorschlägen s. Hope Simpson, ebd., S. 233ff. (Mykene); Mountjoy, in: AnatSt 48, 1998, S. 33ff. (die Dodekanes und die gegenüberliegende westkleinasiatische Küste mit Rhodos als Zentrum).

${ }^{18}$ S. KUB XIV 3.

${ }^{19}$ S. Bryce, in: Oxf]A 8, 1989, S. 300; Gline, International Trade, S. 123f., C.13.

${ }^{20} \mathrm{~S}$. KBo II 11 rev. 11 '-12'.

21 Zaccagnini, in: Rowlands et al., Centre and Periphery, S. 58 und Anm. 41; Cline, International Trade, S. 124, C.14.

22 Liverani, Amarna Essays, S. 25. 
Über solche diplomatischen Geschenke schweigen die Linear B-Archive. Wir erfahren jedoch von kostbaren Gegenständen (Prunkmöbeln, Goldund Silbergefäßen, Schwertern u.a.), die in den Palastmagazinen oder Schatzkammern aufbewahrt wurden.$^{23}$ Es ist sehr wahrscheinlich, daß manche von diesen Preziosen, die regelmäßig inventarisiert wurden, die mykenischen Paläste verließen, um als Geschenke an andere mykenische oder fremde Könige dargebracht zu werden. Diese Vermutung läßt sich nur in einem Fall durch ein interessantes Detail stützen. Eine Anzahl von feinen Gewändern wird in den Täfelchen aus dem Palast von Knossos als xenwia bezeichnet $(\operatorname{Ld}[1]) .{ }^{24}$ Dieser Begriff entstammt dem Wort 乡́ćvos (Fremder, Gast) und dürfte eine ursprüngliche Bedeutung als „dem Gast zugehörig“ gehabt haben. Ob er im Kontext der mykenischen Linear B-Täfelchen in diesem Sinn verwendet wurde oder eine zusätzliche Bedeutungsnuance erlangte, läßt sich nicht sagen. Nach einer plausiblen Vermutung könnten hier feine Gewänder gemeint sein, die entweder als Willkommensgeschenk für fremde Gäste, als Ware für den kommerziellen Export oder als Geschenk für den diplomatischen Geschenkaustausch bestimmt waren. ${ }^{25} \mathrm{Daß}$ ägäische Fürsten dem ägyptischen König derartige Geschenke schickten, läßt sich durch die bekannten Fremdvölkerdarstellungen der thebanischen Privatgräber der 18. Dyn. belegen, in denen gefaltete Stoffe unter anderen kostbaren Gaben abgebildet werden (Abb. 1). ${ }^{26}$ Über diesen möglichen indirekten Hinweis auf Waren, die für einen königlichen Geschenkaustausch bestimmt waren, hinaus haben die mykenischen Archive nichts zu bieten.

Ausgehend vom schriftlich überlieferten Geschenkaustausch zwischen mykenischen Herrschern und ihren orientalischen Partnern, ist es sicherlich legitim zu vermuten, daß einige der zahlreichen Preziosen orientalischer Provenienz aus mykenischen Grab- und Siedlungskontexten als diplomatische Geschenke fremder Könige in die Ägäis gelangten. Ein definitiver Beweis ist allerdings in keinem Fall möglich. Was am ehesten mit einem diplomatischen Geschenkaustausch in Verbindung gebracht werden kann, kam 1963 in einem Raum des sogenannten Palastes von

${ }^{23}$ S. z.B. die pylische Ta-Serie, Killen, ebd. (s.o. Anm. 6), S. 254.

${ }^{24}$ Killen, ebd. (s. Anm. 6), S. 254. 263 mit Anm. 67. Zu dieser Tontafel-Serie s. ausführlich ders., in: Risch/Müllenstein, Colloquium Mycenaeum, S. $151 \mathrm{ff}$.

${ }^{25}$ Killen, ebd. (s. Anm. 6), S. 263.

${ }^{26}$ Wachsmann, Aegeans, S. 75 Taf. 36.37 B. 38 A; Barber, Prehistoric Textiles, S. 335 Abb. 15.19 . 


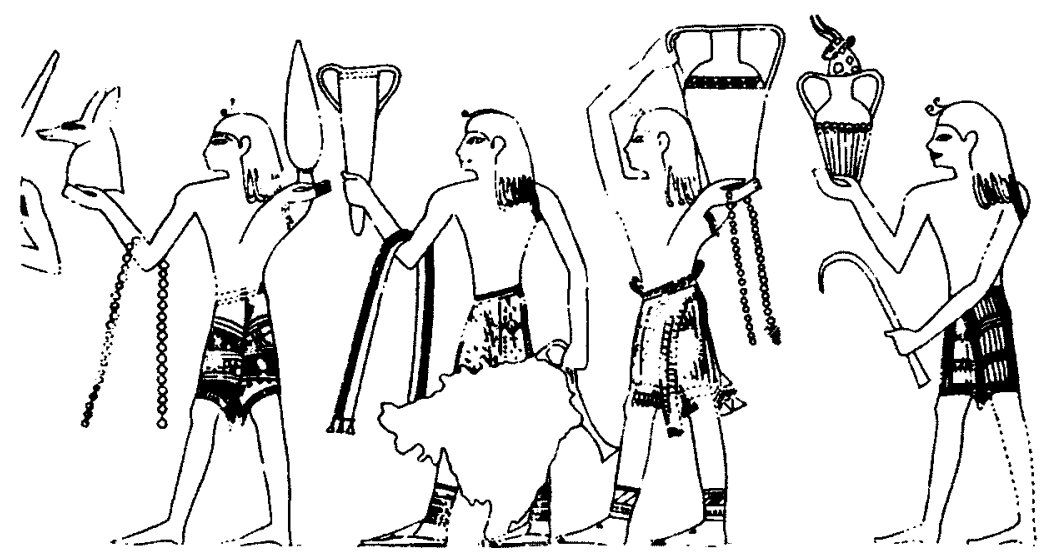

Abb. 1: Ägäische Gabenbringer aus dem thebanischen Grab des Mencheperreseneb (TT 86).

Kadmos in Theben ans Licht. Es handelt sich dabei um eine Gruppe von ca. 50 geschnittenen Rollsiegeln und vorgefertigten Rohlingen, die in ihrer Mehrzahl kassitisch-babylonischer Provenienz sind und bis auf zwei Ausnahmen aus Lapislazuli herausgearbeitet wurden (Taf. XII). ${ }^{27}$ Der Aufmerksamkeit Edith Poradas, die diesen herausragenden Fund veröffentlichte, verdanken wir die Erkenntnis, daß der qualitätvollste Bestandteil dieser Gruppe eine ganze Mine (496 Gramm) Lapislazuli ausmacht, was für die Zusammengehörigkeit der einzelnen Stücke spricht (also eine Sendung). Porada konnte der Versuchung nicht widerstehen, dieses Ensemble mit den in der diplomatischen Korrespondenz genannten Geschenken von Rollsiegeln und Lapislazuli zu verbinden, deren Mengen nach der Ethik des königlichen Geschenkaustausches mit peinlicher Genauigkeit festgehalten wurden, damit der Absender ein im Wert mindestens äquivalentes Geschenk fordern konnte. Poradas provokativer Erklärungsversuch, daß die Rollsiegel Geschenke des Assyrerkönigs Tukulti-Ninurta I. aus seiner in Babylonien gemachte Beute waren, bleibt eine attraktive, jedoch kaum beweisbare Hypothese. Sehr plausibel ist hingegen, daß sie tatsächlich als diplomatisches Geschenk eines assyrischen oder orientalischen Herrschers nach Theben gelangten. Sie könnten dadurch einen willkommenen archäologischen Beweis

${ }^{27}$ Porada, in: AfO 28, 1981/82, S. Iff. 
für die aktive Teilnahme der mykenischen Zentren an der Geschenkdiplomatie jener Zeit liefern.

\section{ABgaben}

Es kann kein Zweifel daran bestehen, daß für die mykenischen Palastzentren die ,Besteuerung' der abhängigen Bevölkerung neben der eigenen Produktion die wichtigste Quelle für die Anhäufung wirtschaftlichen Kapitals war. ${ }^{28}$ Für diese Kategorie ökonomischen Handelns bilden zwar die Linear B-Täfelchen eine weitaus ergiebigere Quelle als es bei den Geschenken der Fall war, doch insgesamt betrachtet bleibt unser Bild vom mykenischen Abgabensystem sehr fragmentarisch. Einen genaueren Einblick in das Konzept der fiskalischen Abschöpfung der nicht-palatialen Produktion erlauben uns nur die relevanten Serien der Tontafeln aus Pylos. Daß die übrigen mykenischen Palastzentren ein ähnliches Besteuerungssystem hatten, ist vor allem aufgrund der Existenz einer gemeinsamen ,Abgabenterminologie' eine legitime Vermutung. In der pylischen Administration kamen hauptsächlich vier Abgabenformen zum Tragen:

a) Abgaben an verschiedenen Naturprodukten, welche die abhängigen Siedlungen als Kollektive leisteten (Ma- und Na-Serien). ${ }^{29}$ Für diese Abgabenform werden in der Regel die Begriffe $d o-k e, a-p u-d o-k e$ und $a-p u-d o-s i$ verwendet. ${ }^{30}$ Als wichtigste Quelle für die Rekonstruktion des mykenischen Abgabensystems gilt die Ma-Serie. ${ }^{31}$ In dieser thematisch homogenen Gruppe von Täfelchen erscheinen als Lieferanten keine Personen, sondern stets Ortschaften, die Abgaben von sechs festgelegten Warensorten in einem proportionalen Verhältnis ablieferten: Tierhäute, Wachs, Textilien (?), Gewürze (?) und zwei weitere nicht näher identifizierbare pflanzliche Produkte. Bis auf eine mögliche Ausnahme (die Textilien) scheint keine der abgegebenen Waren aus ökonomischer Sicht besonders signifikant gewesen zu sein. Ein anderes Steuerkonzept zeigt eine weitere Tafel aus Pylos (Nn 831), die die Abgabe von Leinen seitens

${ }^{28}$ S. Killen, ebd. (s.o. Anm. 6), S. 270 f.

${ }^{29}$ S. Ventris/Chadwick, Documents, S. 213f; de Fidio, in: SMEA 23, 1982, S. 83ff; Killen, ebd. (s.o. Anm. 6), S. 246f; Halstead, in: ProcCambrPhilSoc 38, 1992, S. 59.

3) S.u.

31 de Fidio, ebd. (s.o. Anm. 29), S. 84ff; Perna, in: Laffineur/Nicmeier, Politeia, S. $227 \mathrm{ff}$. 
einer Ortschaft registriert, deren Namen nur fragmentarisch erhalten ist. ${ }^{32}$ Die pylische Bürokratie dringt hier in der Besteuerung des abhängigen Territoriums eine Ebene tiefer ein. Objekte der administrativen Erfassung sind nun einzelne Personen oder Personengruppen dieses Ortes. Was der Anlaß der Lieferung war und wie oft die Palastadministration in den von ihr kontrollierten Ortschaften, wie in diesem Fall, die Ebene des Individuums erreichte, wissen wir nicht.

b) Ablieferungen eines prozentualen Anteils der Ernte von Grundstücken, die Privatpersonen oder dem ,Volk ${ }^{k}$ (/dãmos/) gehörten. ${ }^{33}$ In den meisten Fällen handelte es sich dabei um Parzellen, die von ihrem ,Besitzer' an Dritte verpachtet waren. Dieser Abgabentyp wird entweder als /dosmos/ (für die Grundstücke der Privatpersonen) ${ }^{34}$ oder als wo-ze (für die Grundstücke des /dāmos/) ${ }^{35}$ bezeichnet. ${ }^{36}$ Die relevanten Tontafeln (E-Serie) erwähnen allerdings in allen Fällen nur die Obligation, die mit diesem Land verbunden ist, nicht jedoch die tatsächliche Ablieferung des Produktionsanteils an den Palast.

c) Eine Steuer per capita, die lokalen Würdenträgern und anderen Mitgliedern der bürokratischen Elite auferlegt war, die als Lieferanten von Bronze- oder Goldmengen erscheinen. ${ }^{37}$ Weitere Abgabeleistungen (/dosmoi/), die die Lieferungen von Tieren und landwirtschaftlichen Produkten seitens hoher Palastoffiziere und des /dāmos/ an Poseidon umfassen, sind in ihrer genauen Einordnung in das mykenische Besteuerungssystem problematisch. ${ }^{38}$

d) Obligationen von spezialisierten Handwerkern, die in einem besonderen Abhängigkeitsverhältnis zum Palast standen. ${ }^{39}$

32 Killen, ebd. (s.o. Anm. 6) S. 247. 260; Halstead, in: Voutsaki/Killen, Economy and Politics, S. 44.

${ }^{33}$ S. Heubeck, Frühgriechische Lineartafeln, S. 55ff; Killen, ebd. (s.o. Anm. 6), S. 244ff;;

Deger-Jalkotzy, in: Heltzer/Lipiński, Society and Economy, S. $31 \mathrm{ff}$.

${ }_{34}$ S. de Fidio, Dosmoi.

35 S. Duhoux, Vocabulaire Économique, S. 30f.; Deger-Jalkotzy, ebd., S. 38ff.

${ }^{3 t}$ Die Landbesitzverhältnisse in mykenischer Zeit, eine der Schlüsselfragen der Mykenologie, bleiben in vielen Aspekten dunkel, s. Killen, ebd. (s.o. Anm. 6), S. 243ff.; Hiller/Panagl, Frühgriechische Texte, S. 142ff.; Deger-Jalkotzy, ebd. (s.o. Anm. 33), S. $31 \mathrm{ff}$.

37 S. z.B. Jn 829 .

39 S. de Fidio, Dosmoi, S. 77 ff.

${ }^{39}$ S. Killen, ebd. (s.o. Anm. 6), S. 272f.; ders., in: Voutsaki/Killen, Economy and Politics, S. $161 \mathrm{ff}$. 
$\mathrm{Zu}$ den überlieferten Abgabenleistungen zählen ferner die ökonomischen Verpflichtungen von Personen, die eine besondere wirtschaftliche Beziehung zum Palast unterhielten. Es handelt sich dabei um die Leistungen von ,Mittelsmännern' an die Zentraladministration. Diese Personen, die in der mykenologischen Literatur als owners oder collectors bekannt sind, übernahmen offensichtlich die Verantwortung und damit das finanzielle Risiko eines Bereichs der Palastwirtschaft und waren dem Palast gegenüber zur Leistung eines prozentualen Anteils der Produktion verpflichtet. ${ }^{40}$

Der Versuch, den Charakter der einzelnen mykenischen Abgabeformen durch etymologische Beobachtungen zu erschließen, stößt zunächst auf eine methodische Schwierigkeit. Die genaue inhaltliche Bedeutung mehrerer Begriffe wird in der Regel anhand des semantischen Feldes der Wörter in den späteren griechischen Texten ermittelt. Da es sich allerdings in vielen Fällen um allgemeine Begriffe handelt, ist es durchaus möglich, daß sich ihre semantische Füllung von Zeit zu Zeit geändert hat. Deswegen ist hier bei jeder etymologischen Erklärung Vorsicht geboten. Die Eintreibung der Abgaben in den Linear B-Täfelchen wird durch eine inhaltlich kohärente Gruppe von drei Wörtern registriert: do-ke, $a-p u-d o-k e$ und $a-p u-d o-s i$. Die Deutung aller drei Termini aus derselben Wortfamilie ist in der Etymologisierung des Wortstammes fest verankert. Das Wort $d o-k e$ ist die Aoristform des griechischen $\delta i \delta \omega \mu \mathrm{u}$ (,geben'). Das Wort a-pu-do-ke ist die Aoristform des Verbs $\dot{\alpha} \pi \mathrm{o} \delta \dot{\delta} \delta \omega \mu \mathrm{t}$, (,abgeben $\left.{ }^{6}\right)$. Das Wort $a-p u-d o-s i\left({ }^{*} \alpha \pi v \delta_{\delta o \sigma} \varsigma_{-}\right.$, Abgabe $)$stellt seine Substantivform dar. Eine textbezogene Interpretation von $\delta i \delta \omega \mu \iota$ macht deutlich, daß es kein schlichtes, Geben' ausdrückt, sondern eine Lieferung im Rahmen einer vertraglich festgesetzten Verpflichtung, in anderen Worten: die Begleichung einer ,Schuld ${ }^{6}{ }^{41} \mathrm{Ob}$ der Begriff $a-p u-d o-k e$ eine andere Nuance des mit do-ke beschriebenen Konzepts ausdrückt, läßt sich aufgrund linguistischer oder kontextueller Kriterien nicht

4) S. Bennet, in: Olivier, Mykenaika, S. 65ff; Carlier, in: ebd., S. 159ff.; Driessen, in: ebd., S. 197ff; Godart, in: ebd., S. 257ff.; Killen, in: Laffineur/Niemeier, Politeia, S. $213 \mathrm{ff} ;$ Rougemont, in: dies./Olivier, Épigraphie créto-mycénienne, S. $431 \mathrm{ff}$; dies., in: Voutsaki/Killen, Economy and Politics, S. 129ff.; de Fidio, in: ebd., S. 2 1f. Die Rolle und der Verantwortungsbereich dieser ,Mittelsmänner", die noch nicht eindeutig geklärt sind, dürfte man im Anschluß an eine bewährte orientalische Praxis als ,Palastgeschäft bezeichnen, s. Renger, in: Saeculum 40, 1989, S. 167. 177; ders., in: Orientalia 63, 1994, S. 170. 172ff.

${ }^{41}$ Lejeune, in: MusHelv 32, 1975, S. If: „Le verbe $\delta i ́ \delta \omega \mu$, dans nos textes, signifie partout 's'acquitter d'un dü" ". 
erkennen. ${ }^{42}$ Einstimmig vermuten Lejeune und Duhoux, daß es sich dabei nur um einen stilistisch und nicht inhaltlich bedingten Unterschied handele. Die parallele Verwendung von $a-p u-d o-k e$ (Verb) und $a-p u-d o-s i$ (Substantiv) scheint zunächst aus administrativer Sicht belanglos zu sein, da sich beide offensichtlich auf den gleichen Typ administrativer Handlung bezogen: Sie bezeichnen eine erfolgte Lieferung in Erfüllung einer Obligation im oben beschriebenen Sinne. ${ }^{43}$ Eine Einengung der Bedeutung von $a-p u-d o-s i$ auf ,Kopfsteuer, wie Olivier sie vorschlägt, ist nicht legitim. ${ }^{44}$ Dagegen spricht vor allem die Verwendung des Wortes in den pylischen Täfelchen Fr 1184 und Un 267 im Zusammenhang mit einer Transaktion zwischen zwei Personen. ${ }^{45}$

Neben der etymologischen und textimmanenten Annäherungsweise des mykenischen Abgabensystems ist in den letzten Jahren die Bedeutung einer weiteren Quellengruppe immer deutlicher geworden. Es handelt sich um die Versiegelungen, das zweite Hauptinstrument administrativen Handelns. ${ }^{46}$ Ein verhältnismäßig geringer Teil der ca. 1000 mykenischen Tonplomben ist beschriftet. Die kurzen Inschriften beziehen sich auf die Art, Menge, Herkunft der gesiegelten Waren sowie - und dies ist in unserem Zusammenhang von besonderem Interesse - auf den administrativen Kontext einer Lieferung. Die vorhin erwähnten Termini $d o-k e$, $a-p u-d o-k e$ und $a-p u-d o-s i$ sind dabei mehrfach belegt. Ein interessanter Befund aus dem Archivraum des Palastes von Pylos zeigt, wie diese beiden bürokratischen Instrumente in zwei Phasen ein und derselben administrativen Prozedur eingesetzt wurden. In diesem ,Archivraum“ kam eine mit dem Ideogramm *152 (einem Tierfell) und dem Wort $a-p u-d o-s i$ beschriftete Tonplombe zutage (Taf. XIII), die vom Gegenstand, den sie markierte, bereits abgetrennt war. ${ }^{47}$ Diese Plombe wurde von derselben Person beschriftet, die verantwortlich für die Verfassung der Täfelchen der Ma-Serie war, in der dasselbe Produkt als Abgabeposten auftaucht. Es scheint also, daß die Schreiber der Palastadministration

\footnotetext{
${ }^{42}$ S. hierzu ausführlich Duhoux, in: Minos 9, 1968, S. 10lff.

${ }^{43}$ Lejeune, ebd. (s.o. Anm. 41), S. 3f.: "Lapudosi se situe donc au niveau de l'exécution du contrat, non de sa stipulation. Il s'agit de la fourniture effective (parfaite ou imparfaite) de ce qui esı dû; c'est une donnéc de fait, non une donnée de droil".

"H Olivier, in: Pini, Die Tonplomben, S. 71 Anm. 8.

45 Zu den Tontafeln s. Lejeune, ebd. (s.o. Anm. 41), S. 3.

4t S. zusammenfassend Aravantinos, in: Palaima/Shelmerdine, Pylos Comes Alive, S. 41 ff.; Palaima, ebd. (s.o. Anm. 5), S. 249f.; ders., in: Ferioli et al., Administration, S. $37 \mathrm{ff}$.

"7) Hierzu Palaima, Scribes, S. 61f. (S90-H2); Olivier, in: Pini, cbd. (s.o. Anm. 44), S. 71 f. Kat. Nr. 32 Taf. 13; Palaima, in: Müller, Minoisch-mykenische Siegelglyptik, S. 228.
} 
unter der Anleitung von höher stehenden Beamten ${ }^{48}$ in den jeweiligen Siedlungen die Abgaben sammelten, vor Ort in Bündeln oder Päckchen versiegelten, dann die Lieferungen an den Palast begleiteten, dort die Tonplomben von den Produkten trennten und sie eventuell als Vorlage für das Verfassen der Tontäfelchen nutzten.

Was fehlt in den Tontafeln, die sich auf verschiedene Abgabenformen beziehen? Es fehlt die ökonomische Grundlage jedes antiken Staates, nämlich die massiven Getreidelieferungen. ${ }^{49}$ Getreide als Hauptanbauprodukt, das leicht meßbar, transportierbar und lagerfähig ist, bietet ja eine ideale Abgabenform. In den Linear B-Texten gibt es trotz dieser rätselhaften Informationslücke Hinweise, die die Existenz von palatialen Domänen befürworten. Auf Tafeln der E- und F-Serien aus Knossos werden Getreide und Oliven in Zusammenhang mit dem Eintrag $a-m a$, in einigen Fällen auch mit $e-p i-k e-r e$ kombiniert, was - nach einer sehr plausiblen Vermutung Killens - als /ama epi khereil, "harvest at hand, in hand” im Sinne von „Ernte zur Verfügung“ zu verstehen ist. ${ }^{50}$ Dabei scheint es sich laut Killen nicht um eine Lieferung zu handeln, sondern lediglich um eine Aufnahme der verfügbaren Ernte, die sich auf verschiedene Lagerplätze der Peripherie verteilte. Die Tatsache, daß die Ernte von Getreide und Oliven zu zwei unterschiedlichen Zeitpunkten stattfand, macht den Sinn der Verwendung von e-pi-ke-re nachvollziehbar: Hier geht es nicht um das Abschlußergebnis der diesjährigen Ernte, sondern um einen Bericht zum augenblicklichen Vorrat der Lager. Wenn dieses Fehlen nicht durch den Zeitpunkt der Zerstörung der Paläste bedingt ist, ist es legitim zu vermuten, daß es keine Lieferungen im Rahmen eines Abgabensystems gab. Dies wäre denkbar, wenn Getreide in palatialen Domänen angebaut wurde. Eine solche Form der direkten Bewirtschaftung des wichtigsten agrarischen Produkts läßt sich aus der Sicht der Palastverwaltung sehr gut nachvollziehen. In diesem Fall würde sie das Gesamtprodukt und nicht einen prozentual bestimmbaren Abzug (Abgabe) aus der Produktion von Personen oder Kollektiven ernten. ${ }^{j 1}$

${ }^{48}$ Diese Personen, von denen wir Name und Zuständigkeitsbereich nicht kennen. haben uns auf den Tonplomben ihre, administrativen Fingerabdrücke" hinterlassen gemeint sind hier die Siegelabdrücke.

${ }^{+9}$ S. hierzu de Fidio, ebd. (s.o. Anm. 29), S. 135; Halstead, in: French/Wardle, Greek Prehistory, S. 522.

50 Killen, in: Minos 29-30, 1994-1995, S. 329f.

it Zur wirtschaftlichen Bedeutung von Palastdomanen, die ihren gesamten Ertrag an die Zentraladministration lieferten, s. z.B. Salonen, Agricultura, S. 282ff; Postgate, Early Mesopotamia, S. I69f; Panagiotopoulos, ebd. (s.o. Anm. 12), S. 148. 
Wenn die agrarisch produzierende Bevölkerung nicht mit Abgaben an Getreide fiskalisch abgeschöpft wurde, war sie sicherlich gezwungen, zeitweilig Fronarbeit für die Beackerung der palatialen Domänen zu leisten. Die Frage, warum der Palast die Lieferungen von agrarischen Produkten aus diesem wichtigsten ökonomischen Sektor nicht im Rahmen der Linear B-Administration festhielt, läßt sich vielleicht mit dem oben angesprochenen selektiven Fokus der Tontafeln erklären, der hauptsächlich der Erfüllung von ökonomischen Verpflichtungen seitens der abhängigen Bevölkerung sowie den Ausgaben des Palastes galt. Die Lieferung der Ernte aus den palatialen Domänen, die sich von Beginn an im Besitz des Palastes befand, war keine Transaktion zwischen dem Palast und einem anderen zu Abgaben verpflichteten Partner und mußte somit vermutlich nicht in Form einer Zwangsabgabe registriert werden. ${ }^{52}$

Abschließend muß betont werden, daß die uns verfügbaren Daten kaum quantifizierbar sind, so daß wir keine konkrete Vorstellung davon gewinnen können, welchen Anteil der Produktion der Bevölkerung der Palast sich aneignete. Dennoch steht außer Zweifel, daß die Besteuerung und die mutmaßliche Frondienstpflicht einen schweren Eingriff in das Leben der für die eigene Subsistenz produzierenden Bevölkerung darstellten und somit als Ursache sozialer Spannung neben der ökonomischen auch eine politische Dimension hatten.

\section{Geschenke/Abgaben und die BiLdmedien}

Angesichts der prominenten Stellung von Geschenken, Tributen und Abgaben in der ägyptischen und orientalischen Bildtradition drängt sich auch in unserem Zusammenhang die Frage auf, ob diese Transaktionstypen Eingang in die Bilderwelt der mykenischen Palastzentren gefunden haben. Wurden Geschenke oder Abgaben in den verschiedenen Bildmedien, allen voran den Fresken, thematisiert? Was die Abgaben anbelangt, gibt es keine Belege, daß diese administrative

${ }^{52}$ Eine interessante Parallele liefert der ägyptische Terminus sm.w, der sowohl die Bedeutung von ,Ernte' als auch ,Erntesteuer ' hatte, s. Römer, Gottes- und Priesterherrschaft, S. 378ff. Römer vermutet aufgrund der Wortsemantik ein System der Agrarproduktion, in dem die Produzenten nicht Feldeigentümer, sondern lediglich Beauftragte waren. Die Ablieferung der Getreideproduktion an die staatlichen Scheunen ist daher als „Erfüllung eines Produktionsauftrages und keine Abgabe“ anzusehen. 


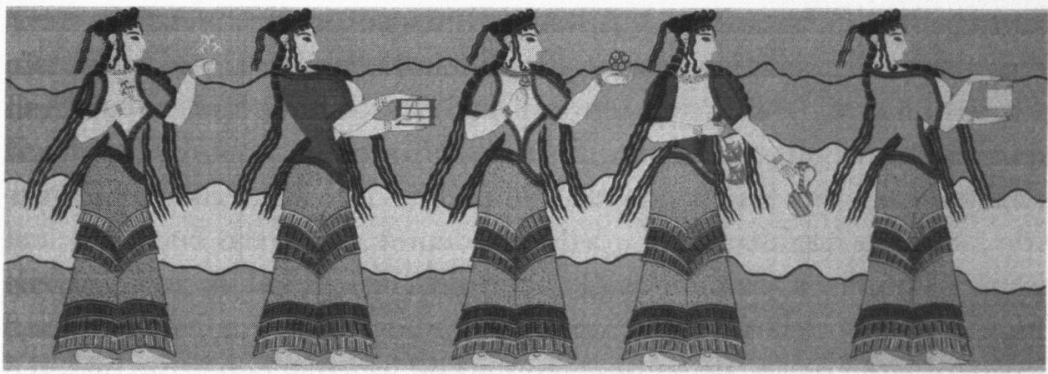

Abb. 2: Prozession von gabenbringenden Frauen. Wandmalerei aus dem mykenischen Palast von Theben (Rekonstruktion H. Reusch).

Prozedur in der höfischen Kunst verewigt wurde. Dies ist sicherlich nicht überraschend, da die Eintreibung der Abgaben offensichtlich als eine rein profane Handlung vonstatten ging, die keinen repräsentativen Wert für die palatiale Elite hatte. Geschenksendungen eines fremden Fürsten oder Königs sind nach unserem jetzigen Kenntnisstand ebenfalls nicht dargestellt. Obwohl die mykenischen Herrscher am Netzwerk der internationalen Geschenkdiplomatie teilhatten und offensichtlich kostbare ausländische Geschenke erhielten, haben diese Geschenksendungen die zeitgenössische Kunst nicht inspiriert. Sollen wir vermuten, daß am Hof eines mykenischen Palastes die Audienz einer fremden Gesandtschaft nicht so glanzvoll inszeniert war wie in Ägypten oder in anderen königlichen Residenzen des östlichen Mittelmeers? Oder ist dieses Fehlen durch die lückenhafte Überlieferungslage zu erklären? Auch in diesem Fall läßt sich keine sichere Antwort geben.

Was wir von den mykenischen Palästen kennen, sind Prozessionen, die aus gabenbringenden Figuren bestehen. Sie kommen in allen vier großen Palästen vor (Mykene, Tiryns, Theben, Pylos) und zeichnen sich durch eine gewisse ,Monumentalität ${ }^{6}$ der Aufführung aus, nämlich ein wandfüllendes Format mit fast lebensgroßen Figuren und ein rigides Darstellungsschema, wonach die Prozessionsteilnehmer gravitätisch und mit klaren Abständen nebeneinander aufgereiht sind (Abb. 2). ${ }^{53}$ Es kann keinen Zweifel daran geben, daß der Anlaß der dargestellten Handlung eine kultische Hofzeremonie war. Den Hauptakzent dieser höfischen Kultvorgänge stellen die kostbaren Gaben dar: Pyxiden,

${ }^{53}$ S. Immerwahr, Aegean Painting, S. 114ff.; Lurz, Der Einfluß Ägyptens, S. 87ff. 
Prunkgefäße, Schmuck u.a. ${ }^{54}$ Auch wenn der zeremonielle Anlaß dieser Prozessionen kein diplomatischer Geschenkaustausch war, ist es möglich, die hier abgebildeten Preziosen mit den Geschenken in Beziehung zu setzen, denn beide gehörten ein und derselben sozioökonomischen Kategorie an. Sie lassen sich am besten mit dem homerischen Begriff von $\kappa \varepsilon \iota \mu \dot{\eta} \lambda_{1} \alpha$ umfassen, der wörtlich bedeutet, etwas, das beiseite gelegt werden kann'. Sie dienten als Symbole des Reichtums oder Ansehens. Wie M. Finley richtig beobachtete, bestand der doppelte Nutzen dieser Kostbarkeiten darin, sie zu besitzen und sie wegzugeben. ${ }^{55}$ Sie bildeten einen Bestandteil des im Sinne Pierre Bourdieus ,symbolischen Kapitals ${ }^{\star}$ des Palastes. ${ }^{56}$ Die Akkumulation ,symbolischen Kapitals' in Form von Ehre und Prestige - erworben z.B. im Fall der mykenischen Paläste durch den Besitz von Prunkobjekten, die Ausrichtung von Festen und durch Schenkungen an Götter, Heiligtümer oder Beamte - erlaubte es, eine pflichtgebundene ,Klientel ${ }^{\natural}$ zu schaffen, die Abgaben lieferte bzw. bei diversen Produktionsprozessen zum Nutzen der Oberschicht als Arbeitskraft eingesetzt werden konnte. Das symbolische Kapital war allem Anschein nach kein totes Kapital, sondern kam unter gewissen zeremoniellen Umständen in Umlauf: Wir dürfen davon ausgehen, daß der Herrscher Geschenke an seine Beamten und andere Herrscher verteilte, an die Götter stiftete und Feste für das Volk organisierte, auch wenn uns nicht jede dieser politischen/zeremoniellen Anstrengungen durch die vorhandenen ikonographischen und epigraphischen Zeugnisse direkt überliefert ist. Bei feierlichen Anlässen wurden solche Gegenstände präsentiert, wie auch die Fresken selbst implizieren, in denen die Frauen die kostbaren Gaben nicht bloß tragen, sondern zur Schau stellen. Hier wird in erster Linie nicht der Kult an sich, sondern der Glanz des Königtums thematisiert, der in Zeremonien und Prunkgegenständen Gestalt annahm. Es ist sehr wahrscheinlich, daß diese Preziosen, bei denen der symbolische Wert wichtiger als der materielle war, nur innerhalb eines zeremoniellen Kreislaufes zirkulierten und nie in kommerzielle Bahnen flossen. Solange es der herrschenden Klasse dadurch gelang, das einseitige Abgabenverhältnis mit Hilfe einer symbolischen oder materiell begrenzten Gegenleistung als eine reziproke Beziehung darzustellen, wäre die Loyalität der Untergebenen sichergestellt gewesen. Wenn die Überlieferungslage uns kein völlig verzerrtes

${ }^{54}$ S. Boulotis, in: Hägg/Marinatos, Function of the Minoan Palaces, S. 145ff.

${ }^{55}$ Finley, Odysseus, S. 61.

56 Bourdieu, in: Annales 32, 1977, S. 405ff. 
Bild von der ursprünglichen bildlichen Ausstattung der mykenischen Paläste bietet, dürfen wir vermuten, daß neben der Macht der Waffen auch der Glanz der Prunkobjekte und Zeremonien die Erhaltung und Reproduktion dieses politischen Systems gewährleistete.

\section{BIBLIOGRAPHIE}

Aravantinos, Vassilis L., The Use of Sealings in the Administration of Mycenaean Palaces, in: T. G. Palaima/S. W. Shelmerdine (eds.), Bylos Comes Alive. Industry and Administration in a Mycenaean Palace, New York 1984, S. 41-48.

Aravantinos, Vassilis, Mycenaean Texts and Contexts at Thebes: The Discovery of New Linear B Archives on the Kadmeia, in: S. Deger-Jalkotzy/S. Hiller/O. Panagl (eds.), Floreant Studia Mycenaea. Akten des X. Internationalen Mykenologischen Colloquiums, Osterreichische Akademie der Wissenschaften, Phil.-Hist. Klasse, Denkschriften 274 (Veröffentlichungen der Mykenischen Kommission 18), Wien 1999, S. 45-78.

Barber, Prehistoric Textiles: Elisabeth J. W. Barber, Prehistoric Textiles. The Development of Cloth in the Neolithic and Bronze Ages with Special Reference to the Aegean, Princeton 1991.

Bartoněk, Handbuch: Antonin Bartoněk, Handbuch des mykenischen Griechisch, Heidelberg 2003.

Bennet, John, „Collectors" or "Owners"? An Examination of their Possible Functions within the Palatial Economy of LM III Crete, in: Jean-Pierre Olivier (ed.), Mykenaika. Actes du IX' Colloque International sur les Textes Mycéniens et Égéens, BCH Suppl. 25, Athen 1992, S. 65-101.

Boulotis, Christos, Nochmals zum Prozessionsfresko von Knossos: Palast und Darbringung von Prestige-Objekten, in: Robin Hägg/Nanno Marinatos (eds.), The Function of the Minoan Palaces. Proceedings of the 4th International Symposium at the Swedish Institute, Stockholm 1987, S. 145-155.

Bourdieu, Pierre, Sur le pouvoir symbolique, in: Annales: Economie, Sociétés, Civilisations 32, 1977, S. 405-411.

Bryce, Trevor R., Ahhiyawans and Mycenaeans - An Anatolian Viewpoint, in: OxfJA 8, 1989, S. $297-310$.

Carlier, Pierre, Les Collecteurs Sont-ils des Fermiers?, in: Jean-Pierre Olivier (ed.), Mykenaika. Actes du IX Colloque International sur les Textes Mycéniens et Égéens, BCH Suppl. 25, Athen 1992, S. 159-166.

Carruba et al., Studi Orientalistici: Onofrio Carruba/Mario Liverani/Carlo Zaccagnini (eds.), Studi Orientalistici in Ricordo di Franco Pintore, Pavia 1983.

Cline, International Trade: Eric Cline, Sailing the Wine-Dark Sea. International Trade and the Late Bronze Age Aegean, BAR International Series 591, Oxford 1994.

Davies/Duhoux, Linear B: Anna Morpurgo Davies/Yves Duhoux (eds.), Linear B: a 1984 survey. Proceedings of the Mycenaean Colloquium of the VIIIth Congress of the International Federation of the Societies of Classical Studies (Dublin, 27th August 1st September 1984), Bibliothèque des Cahiers de l'Institut de Linguistique de Louvain 26, Louvain-la-Neuve 1985.

De Fidio, Pia, I dosmoi pilii a Poseidon: Una terra sacra di età micenea, in: Incunabula Greca 65, Roma 1977.

De Fidio, Pia, Fiscalità, redistribuzione, equivalenze: per una discussione sull'economia micenea, in: SMEA 23, 1982, S. 83-136.

De Fidio, Pia, Centralization and its Limits in the Mycenean Palatial System, in: S. Voutsakis/ J. Killen, Economy and Politics in the Mycenean Palace States. Proceedings of a Conference held in the Faculty of Classics, Cambridge, Cambridge 2001, S. 15-24.

Deger-Jalkotzy, Sigrid, Landbesitz und Sozialstruktur im mykenischen Staat von Bylos, in: 
M. Heltzer/E. Lipiński (eds.), Society and Economy in the Eastern Mediterranean (c. 15001000 B.C.). Proceedings of the International Symposium Held at the University of Haifa, OLA 23, Leuven 1988, S. 31-52.

Deger-Jalkotzy et al., Floreant Studia Mycenaea: Sigrid Deger-Jalkotzy/Stefan Hiller/Oswald Panagl (eds.), Floreant Studia Mycenaea. Akten des X. Internationalen Mykenologischen Colloquiums in Salzburg vom 1.-5. Mai 1995, Österreichische Akademie der Wissenschaften, Philosophisch-Historische Klasse, Denkschriften 274 (Veröffentlichungen der Mykenischen Kommission 18), Wien 1999.

Driessen, Jan, „Collector's Items”. Observations sur l'Elite Mycénienne de Cnossos, in: JeanPierre Olivier (ed.), Mykenaika. Actes du $\mathrm{DX}^{\mathrm{e}}$ Colloque International sur les Textes Mycéniens et Égéens, BCH Suppl. 25, Athen 1992, S. 197-214.

Driessen, Jan, Data Storage for Reference and Prediction at the Dawen of Curilization? A Review Article with Some Observations on Archives Before Writing, in: Minos 29-30, 1994-95, S. 239-256.

Driessen, Jan, The Northern Entrance Passage at Knossos. Some Preliminary Observations on its Potential Role as „Central Archives”, in: S. Deger-Jalkotzy/S. Hiller/O. Panagl (eds.), Floreant Studia Mycenaea. Akten des X. Internationalen Mykenologischen Colloquiums, Osterreichische Akademie der Wissenschaften, Phil.-Hist. Klasse, Denkschriften 274 (Veröffentlichungen der Mykenischen Kommission 18), Wien 1999, S. 205-226.

Driessen, Chariot Tablets: Jan Driessen, The Scribes of the Room of the Chariot Tablets at Knossos. Interdisciplinary Approach to the Study of a Linear B Deposit, Minos Suppl. 15, Salamanca 2000.

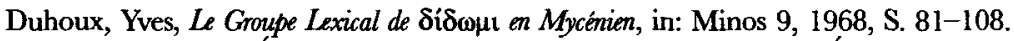

Duhoux, Vocabulaire Économique: Yves Duhoux, Aspects du Vocabulaire Économique Mycénien (Cadastre - Artisanat - Fiscalité), Amsterdam 1976.

Ferioli et al., Administration: Piera Ferioli/Enrica Fiandra/Gian Giacomo Fissore (eds.), Administration in Ancient Societies. Proceedings of Session 218 of the 13th International Congress of Anthropological and Ethnological Sciences, Mexico City, July 29August 5, 1993, Torino 1996.

Finley, Odysseus: Moses I. Finley, The World of Odysseus, London $1977^{2}$.

French/Wardle, Greek Prehistory: Elisabeth B. French/Kenneth Anthony Wardle (eds.), Problems in Greek Prehistory. Papers Presented at the Centenary Conference of the British School of Archaeology at Athens, Manchester April 1986, Bristol 1988.

Godart, Louis, Les Collecterers dans le Monde Egéen, in: Jean-Pierre Olivier (ed.), Mykenaika. Actes du IX'Colloque International sur les Textes Mycéniens et Égéens, BCH Suppl. 25, Athen 1992, S. 257-283.

Hägg/Marinatos, Function of the Minoan Palaces: Robin Hägg/Nanno Marinatos (eds.), The Function of the Minoan Palaces. Proceedings of the Fourth International Symposium at the Swedish Institute in Athens, 10-16 June, 1984, Stockholm 1987.

Hänsel, Handel, Tausch und Verkehr. Bernhard Hänsel (ed.), Handel, Tausch und Verkehr im Bronze- und Früheisenzeitlichen Südosteuropa, Südosteuropa-Schriften 17 (Prähistorische Archäologie in Südosteuropa 11), München/Berlin 1995.

Halstead, Paul, On Redistribution and the Origin of Minoan-Mycenaean Palace Economies, in: E. B. French/K. A. Wardle (eds.), Problems in Greek Prehistory. Papers Presented at the Centenary Conference of the British School of Archaeology at Athens, Bristol 1988, S. 519-529.

Halstead, Paul, The Mycenaean Palatial Economy: Making the Most of the Gaps in the Evidence, in: ProcCambrPhilSoc 38, 1992, S. 57-86.

Halstead, Paul, Mycenean Wheat, Flax and Sheep: Palatial Intervention in Farming and its Implications for Rural Society, in: S. Voutsakis/J. Killen, Economy and Politics in the Mycenean Palace States. Proceedings of a Conference held in the Faculty of Classics, Cambridge, Cambridge 2001, S. 38-50.

Heltzer/Lipiński, Society and Economy: Michael Heltzer/E. Lipiński (eds.), Society and Economy in the Eastern Mediterranean (c. 1500-1000 B.C.). Proceedings of the International Symposium Held at the University of Haifa from the 28th of April to the 2nd of May 1985, OLA 23, Leuven 1988. 
Heubeck, Frühgriechische Lineartafeln: Alfred Heubeck, Aus der Welt der frühgriechischen Lineartafeln. Eine kurze Einfuhrung in Grundlagen, Aufgaben und Ergebnisse der Mykenologie, Göttingen 1966.

Heubeck, Schrift: Alfred Heubeck, Schrift, ArchHom 3, Göttingen 1979.

Hiller/Panagl, Frühgriechische Texte: Stefan Hiller/Oswald Panagl, Die frühgriechischen Texte aus mykenischer Zeit. Zur Efforschung der Linear B-Tafeln, Darmstadt $1986^{2}$.

Hope Simpson, Richard, The Dodecanese and the Ahhiyawa question, in: BSA 98, 2003, S. 203-237.

Ilievski/Crepajac, Tractata Mycenaea: Petar H. Ilievski/L. Crepajac (eds.), Tractata Mycenaea. Proceedings of the Eighth International Colloquium on Mycenaean Studies Held in Ohrid, 15-20 September 1985, Skopje 1987.

Immerwahr, Aegean Painting. Sara A. Immerwahr, Aegean Painting in the Bronze Age, University Park, Pennsylvania 1990.

KBo: Bedrich Hrozný, Hethitische Keilschriftexte aus Boghazköi in Umschrift, mit Übersetzung und Kommentar, Leipzig 1919.

Killen, John, The Knossos Ld(I) Tablets, in: E. Risch/H. Müllenstein (cds.), Colloquium Mycenaeum. Actes du $6^{\text {ème }}$ Colloque International sur les Textes Mycéniens et Égéens Tenu à Chaumont sur Neuchâtel, Neuchâtel 1979, S. 151-181.

Killen, John, The Linear B Tablets and the Mycenaean Economy, in: A. M. Davies/Y. Duhoux (eds.), Linear B: a 1984 survey. Proceedings of the Mycenaean Colloquium of the VIIIth Congress of the International Federation of the Societies of Classical Studies, Louvain-la-Neuve 1985, S. 241-305.

Killen, John, a-ma e-pi-ke-re, in: Minos 29-30, 1994-1995, S. 329-333.

Killen, John, Some Further Thoughts on 'Collectors', in: R. Laffineur/W.-D. Dietrich Niemeier (eds.), POLITEIA. Society and State in the Aegean Bronze Age. Proceedings of the 5th International Aegean Conference, Liège/Austin 1995, S. 213-221.

Killen, John, Some Thoughts on ta-ra-si-ja, in: S. Voutsaki/J. Killen (eds.), Economy and Politics in the Mycenaean Palace States. Proceedings of a Conference held in the Faculty of Classics, Cambridge, Cambridge 2001, S. 161-180.

KUB: Keilschrifturkunden aus Boghazköi.

Laffineur/Niemeier, Politeia: Robert Laffineur/Wolf-Dietrich Niemeier (eds.), POLITELA. Society and State in the Aegean Bronze Age. Proceedings of the 5th International Aegean Conference, University of Heidelberg, Archäologisches Institut 10-13 April 1994, Aegeaum 12, Liège/Austin 1995.

Latacz, Troia und Homer: Joachim Latacz, Troia und Homer. Der Weg zur Lösung eines alten Rätsels, München/Berlin 2001.

Latacz, Homer-Forschung. Joachim Latacz (ed.), Zweihundert Jahre Homer-Forschung. Rückblick und Ausblick, Stuttgart/Leipzig 1991.

Lehmann, Gustav Adolf, Die ,politisch-histonischen'Beziehungen der Agäis-Welt des 15.-13. Th. s v.Chr. zu Agypten und Vorderasien: einige Hinweise, in: Joachim Latacz (ed.), Zweihundert Jahre Homer-Forschung. Rückblick und Ausblick, Stuttgart/Leipzig 1991, S. 105-126.

Lejeune, Michel, $\triangle O \Sigma M O \Sigma$ et AПY $\triangle O \Sigma I \Sigma$, in: MusHelv 32, 1975, S. 1-11.

Liverani, Amarna Essays: Mario Liverani, Three Amarna Essays, Monographs on the Ancient Near East 1/5, Malibu 1979.

Lurz, Der Einfluß Ägyptens: Norbert Lurz, Der Einfluß Ägyptens, Vorderasiens und Kretas auf die Mykenischen Fresken. Studien zum Ursprung der Frühgriechischen Malerei, Frankfurt 1994.

Mountjoy, Penelope, The East Aegean-West Anatolian Interface in the Late Bronze Age: Mycenaeans and the Kingdom of Ahhijawa, in: AnatSt 48, 1998, S. 3367.

Müller, Minoisch-mykenische Siegelglyptik. W. Müller (ed.), Minoisch-mykenische Siegelglyptik. Stil Ikonographie, Funktion. Internationales Siegel-Symposium, Marburg, CMS Beih. 6 , Berlin 2000.

Niemeier, Wolf-Dietrich, Hattusa und Ahhijawa im Konfikt um Millawanda/Milet. Die politische und kulturelle Rolle des mykenischen Griechenland in Westkleinasien, in: Die Hethiter, S. 294-299. 
Olivier, Mykenaïka: Jean-Pierre Olivier (ed.), Mykenaikka. Actes du IX Colloque International sur les textes mycéniens et Égéens organisé par le Centre de l'Antiquité Grecque et Romaine de la Fondation Hellénique des Recherches Scientifiques et l'École Française d'Athènes (Athènes, 2-6 Octobre 1990), BCH Suppl. 25, Athen 1992.

Olivier, Jean-Pierre, El comercio micénico desde la documentación epigráfica, in: Minos 31-32, 1996-1997, S. 275-292.

Olivier, Jean-Pierre, Die beschrifteten Tonplomben, in: Ingo Pini (ed.), Die Tonplomben aus dem Nestorpalast von Pylos, Mainz 1997, S. 70-81.

Palaima, Thomas G., Mycenaean Seals and Sealings in their Economic and Administrative Contexts, in: P. H. Ilievski/L. Crepajac (eds.), Tractata Mycenaea. Proceedings of the 8th International Colloquium on Mycenaean Studies Held in Ohrid, Skopje 1987, S. 249-266.

Palaima, Thomas G., The Scribes of Bylos, Incunabula Graeca 87, Roma 1988.

Palaima, Thomas G., The Last Days of the Bylos Polity, in: R. Laffineur/W.-D. Niemeier (eds.), POLITELA. Society and State in the Aegean Bronze Age. Proceedings of the 5th International Aegean Conference, Liège/Austin 1995, S. 623-632.

Palaima, Thomas G., Sealings as Links in an Administrative Chain, in: P. Ferioli/E. Fiandra/ G.G. Fissore (eds.), Administration in Ancient Societies. Proceedings of Session 218 of the 13th International Congress of Anthropological and Ethnological Sciences, Torino 1996, S. 37-66.

Palaima, Thomas G., The Palaeography of Mycenaean Inscribed Sealings from Thebes and Bylos, Their Place Within the Mycenaean Administrative System and Their Links with the Extra-Palatial Sphere, in: W. Müller (ed.), Minoisch-mykenische Siegelglyptik. Stil, Ikonographie, Funktion. Internationales Siegel-Symposium, Marburg - Berlin 2000, S. 219-238.

Palaima/Shelmerdine, Pylos Comes Alive: Thomas G. Palaima/Synthia W. Shelmerdine (eds.), Pylos Comes Alive. Industry and Administration in a Mycenaean Palace, New York 1984.

Panagiotopoulos, Diamantis, Tributabgaben und Huldigungsgeschenke aus der Levante. Die ägyptische Nordexpansion in der 18. Dynastie aus strukturgeschichtlicher Sicht, in: ÄL 10, 2000, S. $139-158$.

Panagl, Oswald, Handel, Händler und Verkehr im Spiegel griechischer Texte von Linear B bis Homer, in: B. Hänsel (ed.), Handel, Tausch und Verkehr im Bronze- und Früheisenzeitlichen Südosteuropa, München-Berlin 1995, S. 49-52.

Perna, Massimo, Le tavolette della seria Ma di Pilo, in: R. Laffineur/W.-D. Niemeier (eds.), POLITELA. Society and State in the Aegean Bronze Age. Proceedings of the 5th International Aegean Conference, Liège/Austin 1995, S. 227-232.

Pini, Die Tonplomben: Ingo Pini (ed.), Die Tonplomben aus dem Nestorpalast von Pylos, Mainz 1997.

Porada, Edith, The Cylinder Seals Found at Thebes in Boeotia, in: AfO 28, 1981/1982, S. 1-70.

Postgate, Early Mesopotamia: John Nicholas Postgate, Early Mesopotamia. Society and Economy at the Dawn of History, London 1992.

Renger, Johannes, Probleme und Perspektiven einer Wirtschaftsgeschichte Mesopotamiens, in: Saeculum 40, 1989, S. 166-178.

Renger, Johannes, On Economic Structures in Ancient Mesopotamia, in: Orientalia 63, 1994, S. $157-208$.

Risch/Müllenstein, Colloquium Mycenaeum: Ernst Risch/Hugo Müllenstein (eds.), Colloquium Mycenaeum. Actes du Sixième Colloque International sur les Textes Mycéniens et Égéens Tenu à Chaumont sur Neuchâtel du 7 au 13 Septembre 1975, Neuchâtel 1979.

Römer, Gottes- und Priesterherrschaft: Malte Römer, Gottes- und Priesterherrschaft in Agypten am Ende des Neuen Reiches. Ein religionsgeschichtliches Phänomen und seine sozialen Grundlagen, ÄAT 21, Wiesbaden 1994.

Rowlands et al., Centre and Peniphery: Michael Rowlands/Mogens Larsen/Kristian Kristiansen (eds.), Centre and Periphery in the Ancient World, Cambridge 1987. 
Rougemont, Françoise, Quelques problèmes liés à l'étude des „collecteur" dans les tablettes en linéaire B, in: F. Rougemont/J.-P. Olivier (eds.), Recherches récentes en épigraphie crétomycénienne, BCH 122, 1998, S. 431 434.

Rougemont, Françoise, Some Thoughts on the Identification of the "Collectors" in the Linear $B$ Tablets, in: S. Voutsaki/J. Killen (eds.), Economy and Politics in the Mycenaean Palace States. Proccedings of a Conference held in the Faculty of Classics, Cambridge, Cambridge 2001, S. 129-138.

Rougemont/Olivier, Epigraphie créto-mycénienne: Francoise Rougemont/Jean-Pierre Olivier (eds.), Recherches récentes en épigraphie créto-mycénienne, BCH 122, 1998.

Salonen, Agricultura: Armas Salonen, Agricultura Mesopotamica nach sumerisch-akkadischen Quellen. Eine lexikalische und kulturgeschichtliche Untersuchung, Helsinki 1968.

Urk. IV: K. Sethe, Urkunden des ägyptischen Altertums, Bd. 4: Urkunden des Neuen Reichs, Berlin-Graz 1961 (Nachdruck der 2. Auflage).

Ventris/Chadwick, Documents: Michael Ventris/John Chadwick, Documents in Mycenaean Greek, Cambridge $1973^{2}$.

Voutsaki/Killen, Economy and Politics: Sofia Voutsaki/John Killen (eds.), Economy and Politics in the Mycenaean Palace States. Proceedings of a Conference held on 1-3 July 1999 in the Faculty of Classics, Cambridge, Cambridge Philological Society Suppl. 27, Cambridge 2001.

Wachsmann, Aegeans: Shelley Wachsmann, Aegeans in the Theban Tombs, OLA 20, Leuven 1987.

Wagner-Hasel, Der Stoff der Gaben: Beate Wagner-Hasel, Der Stoff der Gaben. Kultur und Politik des Schenkens und Tauschens im archaischen Griechenland, Frankfurt/New York 2000.

Die Hethiter. Helga Willinghöfer/Uta Hasekamp (eds.), Die Hethiter und ihr Reich. Das Volk der 1000 Götter, Bonn 2002.

Zaccagnini, Scambio dei doni: Carlo Zaccagnini, Lo scambio dei doni nel Vicino Oriente durante $i$ secoli $X V$-XIII, Roma 1973.

Zaccagnini, Carlo, On Gift Exchange in the Old Babylonian Period, in: O. Carruba/ M. Liverani/C. Zaccagnini (eds.), Studi Orientalistici in Ricordo di Franco Pintore, Pavia 1983 , S. $189-253$.

Zaccagnini, Carlo, Aspects of Ceremonial Exchange in the Near East during the Late Second Millennium B.C., in: M. Rowlands/M. Larsen/K. Kristiansen (eds.), Centre and Periphery in the Ancient World, Cambridge 1987, S. 57-65. 

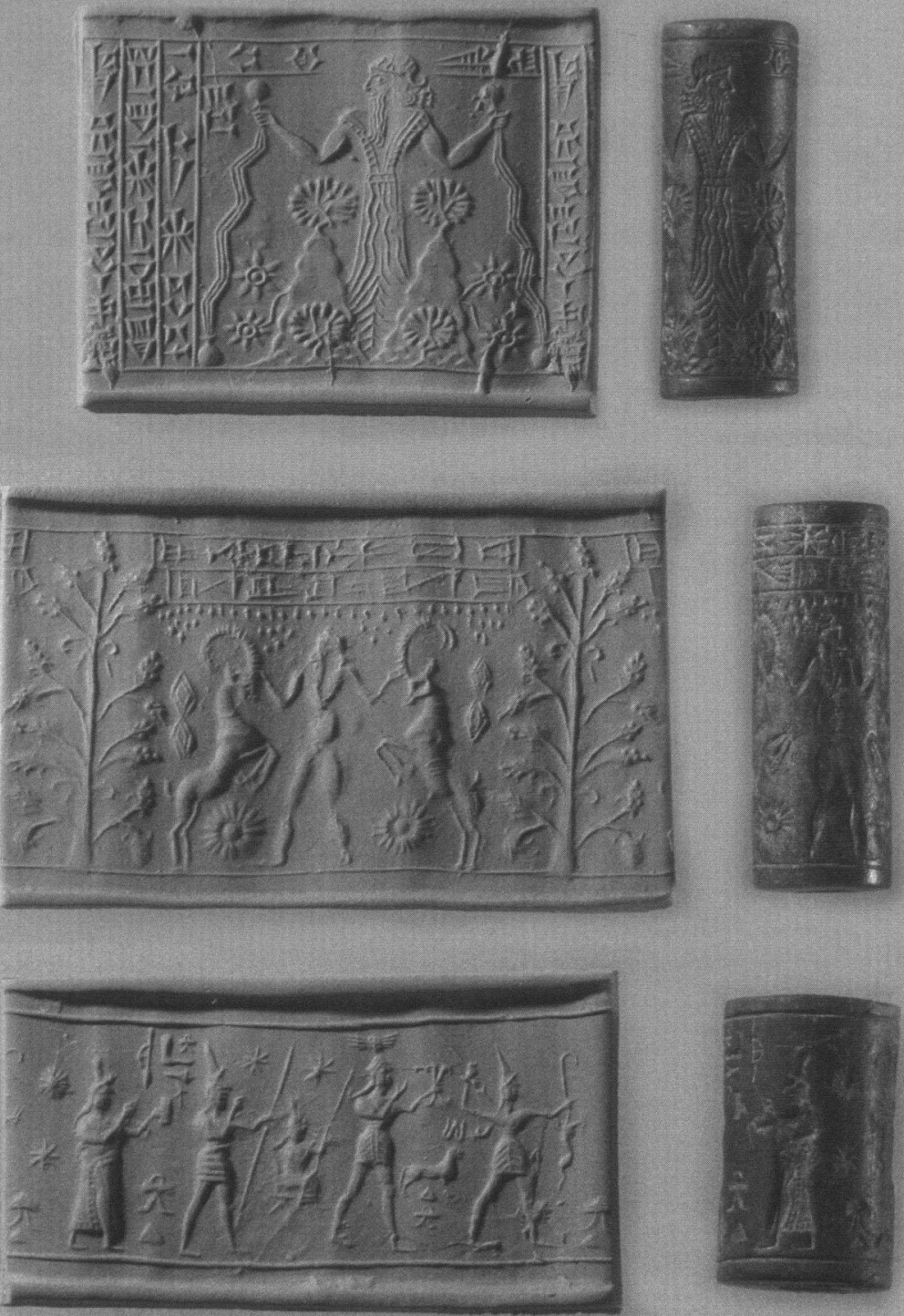

Drei orientalische Rollsiegel aus dem mykenischen Palast von Theben 


\section{Diamantis Panagiotopoulos}

TAFEL XIII

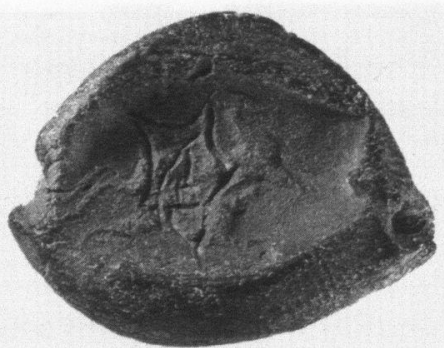

32

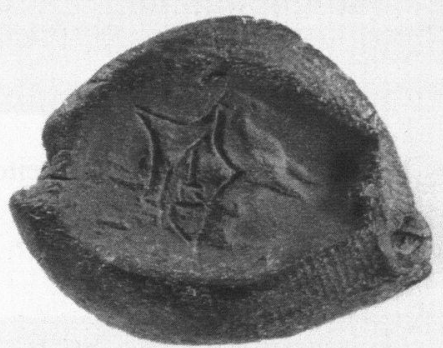

32

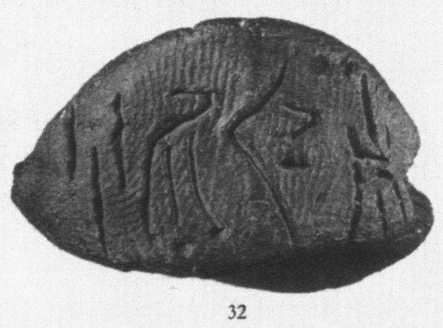

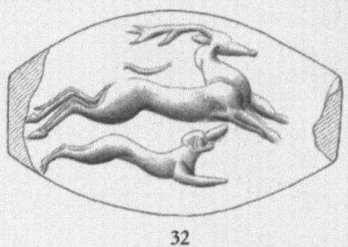
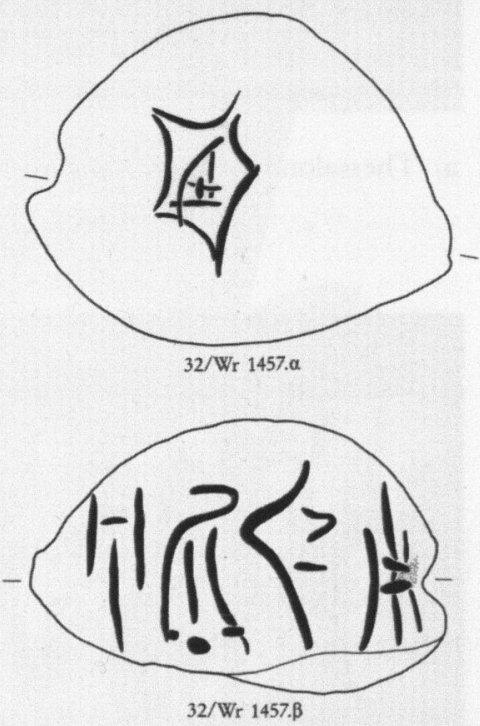

Beschriftete Tonplomben aus dem Archivraum des mykenischen Palastes von Pylos 